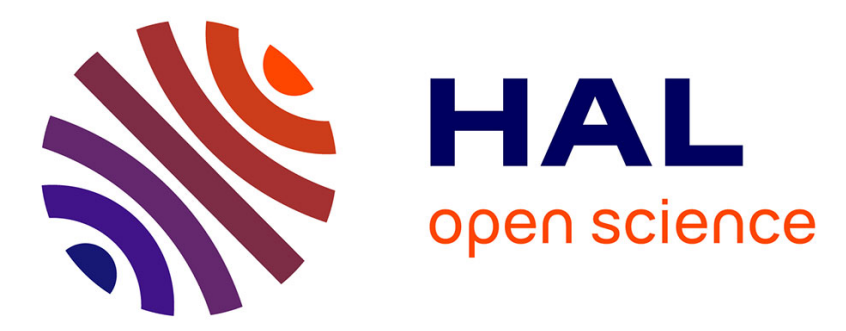

\title{
Refined Sobolev inequalities in Lorentz spaces
} Hajer Bahouri, Cohen Albert

\section{- To cite this version:}

Hajer Bahouri, Cohen Albert. Refined Sobolev inequalities in Lorentz spaces. Journal of Fourier Analysis and Applications, 2011, 17 (4), pp.662-673. hal-00795960

\section{HAL Id: hal-00795960 https://hal.science/hal-00795960}

Submitted on 1 Mar 2013

HAL is a multi-disciplinary open access archive for the deposit and dissemination of scientific research documents, whether they are published or not. The documents may come from teaching and research institutions in France or abroad, or from public or private research centers.
L'archive ouverte pluridisciplinaire HAL, est destinée au dépôt et à la diffusion de documents scientifiques de niveau recherche, publiés ou non, émanant des établissements d'enseignement et de recherche français ou étrangers, des laboratoires publics ou privés. 


\title{
Refined Sobolev inequalities in Lorentz spaces
}

\author{
Hajer Bahouri and Albert Cohen
}

March 1, 2013

\begin{abstract}
We establish refined Sobolev inequalities between the Lorentz spaces and homogeneous Besov spaces. The sharpness of these inequalities is illustrated on several examples, in particular based on non-uniformly oscillating functions known as chirps. These results are also used to derive refined Hardy inequalities.
\end{abstract}

\section{Introduction}

The Sobolev inequality states that for any $0<s<\frac{d}{2}$ there exists a constant $C>0$ such that for any function $f$ defined on $\mathbb{R}^{d}$,

$$
\|f\|_{L^{p}} \leq C\|f\|_{\dot{H}^{s}},
$$

with $\frac{1}{p}:=\frac{1}{2}-\frac{s}{d}$ (therefore $p$ is strictly between 2 and $\infty$ ). A refined version of this inequality was proved in $[10]$ :

$$
\|f\|_{L^{p}} \leq C\|f\|_{\dot{H}^{s}}^{1-\frac{2 s}{d}}\|f\|_{\dot{B}_{\infty, \infty}^{s-\frac{d}{2}}}^{\frac{2 s}{d}},
$$

where $\dot{B}_{\infty, \infty}^{s-\frac{d}{2}}$ is the homogeneous Besov space. Similar results also hold when the smoothness of the function is measured in $L^{q}$ for $1 \leq q<\infty$ : for any $0<s<\frac{d}{q}$,

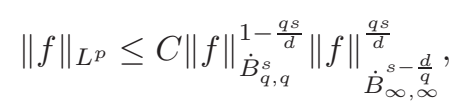

with $\frac{1}{p}:=\frac{1}{q}-\frac{s}{d}$, or the same kind of inequalities with $\dot{W}^{s, q}$ in place of $\dot{B}_{q, q}^{s}$. We also refer to $[3,4,13,14,16]$ for similar results, involving in particular the space $B V$.

These inequalities are generalizations of the usual Sobolev embedding in Lebesgue spaces, their additional feature being that they are invariant under oscillations and fractal transforms see [1]. A significant application is the description of defect of compactness in Sobolev embedding (see [9] in the $L^{2}$ framework and [11] in $L^{p}$ framework).

On the other hand, it is also known that Sobolev and Besov spaces also embed in Lorentz spaces $L^{p, q}\left(\mathbb{R}^{d}\right)$ : with $\frac{1}{p}=\frac{1}{q}-\frac{s}{d}$, one has

$$
\|f\|_{L^{p, q}} \leq C\|f\|_{\dot{B}_{q, q}^{s}} .
$$

This may be derived from the standard inequalities in $L^{p}$ spaces by a real interpolation argument. This justifies looking for refined inequalities of the type (1.1) with $L^{p, q}\left(\mathbb{R}^{d}\right)$ in place of $L^{p}\left(\mathbb{R}^{d}\right)$.

In this paper, such inequalities will be established. The "price to pay" will be a slight modification in one index of the Besov space of negative smoothness, namely

$$
\|f\|_{L^{p, q}} \leq C\|f\|_{\dot{B}_{q, q}^{s}}^{1-\frac{q s}{d}}\|f\|_{\substack{\frac{q s}{d} \\ \dot{B}_{\infty, q}^{s-\frac{d}{q}}}},
$$

for the same range of indices $(s, p, q)$ as in (1.1). It will be shown that this change is unavoidable and that such inequalities are sharp.

The structure of the paper is the following. First, in $\S 2$, we briefly recall some facts on Lorentz and Besov spaces, we establish estimates for Besov spaces which are used in the sequel. In $\S 3$ we give the 
proof of the refined Sobolev inequalities (1.3), which follows from the same line of reasoning as in [10] or [13]. The sharpness of the inequality is discussed in $\S 4$. As with the improved Sobolev inequality (1.1), these inequalities remain sharp for oscillatory functions, but more interestingly the necessity of the above mentioned change of index can be proved by considering non-uniformly oscillating functions known as chirps. Such functions have been deeply studied by Meyer and Jaffard, see [12, 16] and arise naturally in signal processing. Finally, as a by-product of (1.3) we obtain in $§ 5$ a refined version of the Hardy inequalities in the $L^{q}$ framework, namely

$$
\left(\int \frac{|f(x)|^{q}}{|x|^{s q}}\right)^{\frac{1}{q}} \leq C\|f\|_{\dot{B}_{q, q}^{s} \frac{q s}{d}}^{1-}\|f\|_{\substack{\dot{B}_{\infty, q}^{s-\frac{d}{q}} \\ \frac{q s}{d}}}^{.}
$$

A similar inequality was derived in [1] in the case $q=2$. However, our method of proof is significantly simpler and applies to arbitrary values of $q$. Let us stress that Hardy inequalities are of important use in analysis: among other applications, we can mention blow-up methods or the study of pseudo-differentional operators with singular coefficients.

Acknowledgement: the authors are grateful to the anonymous reviewers for their constructive suggestions in improving the paper.

\section{Lorentz spaces and Besov spaces}

For any measurable function $f$ on $\mathbb{R}^{d}$, we define its distribution and rearrangement functions

$$
\mu_{f}(t):=\mid\{x ;|f(x)| \geq t\} \text { and } f^{*}(s):=\inf \left\{t ; \mu_{f}(t) \leq s\right\}
$$

For $1 \leq p<\infty$ and $1 \leq q \leq \infty$, the Lorentz space $L^{p, q}\left(\mathbb{R}^{d}\right)$ is defined as the set of all measurable functions $f$ such that the function $s^{\frac{1}{p}} f^{*}(s)$ belongs to $L^{q}\left(\mathbb{R}_{+}, \frac{d s}{s}\right)$. The $L^{p, q}$ quasi-norm is defined by

$$
\|f\|_{L^{p, q}}:=\left(\int_{0}^{+\infty}\left(s^{\frac{1}{p}} f^{*}(s)\right)^{q} \frac{d s}{s}\right)^{\frac{1}{q}},
$$

with the standard modification when $q=\infty$ which corresponds to the weak $L^{p}$ space $w L^{p}=L^{p, \infty}$. It is well known that $L^{p, p}=L^{p}$ and that Lorentz spaces can be derived from $L^{p}$ spaces by the real interpolation method. In particular, when $1<p<\infty$ we have $L^{p, q}=\left[L^{1}, L^{\infty}\right]_{\theta, q}$ with $\frac{1}{p}=1-\theta$. We refer to $[5,2]$ for these classical results.

In this paper, we shall use the expression of the $L^{p, q}$ quasi-norm in terms of the distribution function $\mu_{f}$, namely

$$
\|f\|_{L^{p, q}}=\left(p \int_{0}^{+\infty}\left(t \mu_{f}(t)^{\frac{1}{p}}\right)^{q} \frac{d t}{t}\right)^{\frac{1}{q}} .
$$

To derive this expression, one first writes

$$
f^{*}(s)^{q}=q \int_{0}^{f^{*}(s)} t^{q-1} d t
$$

so that

$$
\begin{aligned}
\|f\|_{L^{p, q}}^{q} & =\int_{0}^{+\infty} s^{\frac{q}{p}-1} f^{*}(s)^{q} d s \\
& =q \int_{0}^{+\infty} s^{\frac{q}{p}-1}\left[\int_{0}^{f^{*}(s)} t^{q-1} d t\right] d s \\
& =q \int_{0}^{+\infty} t^{q-1}\left[\int_{0}^{\mu_{f^{*}}(t)} s^{\frac{q}{p}-1} d s\right] d t \\
& =p \int_{0}^{+\infty} t^{q-1} \mu_{f^{*}}(t)^{\frac{q}{p}} d t
\end{aligned}
$$

Observing that $\mu_{f}=\mu_{f *}$ we obtain (2.6).

There exists several equivalent definitions of Besov spaces either based on Littlewood-Paley decompositions, moduli of smoothness, approximation procedures, wavelet decompositions. We refer to [18] and [7] for a general treatment, and only recall here the definition based on Littlewood-Paley theory. 
Let $\varphi$ be a function of $d$ variables such that its Fourier transform $\hat{\varphi}$ is non-negative, $C^{\infty}$, supported in the ball $\{|\omega| \leq M\}$ for some $M>1$ and such that $\hat{\varphi}(\omega)=1$ if $|\omega| \leq 1$. For $j \in \mathbb{Z}$ we define the operator $S_{j}$ acting on tempered distributions of $d$ variables by

$$
S_{j} f=2^{d j} \varphi\left(2^{j} \cdot\right) * f
$$

and $\Delta_{j}=S_{j+1}-S_{j}$ or equivalently

$$
\Delta_{j} f=2^{d j} \psi\left(2^{j} \cdot\right) * f,
$$

with $\hat{\psi}(\omega)=\hat{\varphi}\left(\frac{\omega}{2}\right)-\hat{\varphi}(\omega)$. For $s<\frac{d}{p}$ and $q \geq 1$, we define the homogeneous Besov space $\dot{B}_{p, q}^{s}\left(\mathbb{R}^{d}\right)$ as the space of tempered distributions $f$ such that $\sum_{j=-J}^{J} \Delta_{j} f$ converges towards $f$ in $\mathcal{S}^{\prime}$ as $J \rightarrow+\infty$ and such that

$$
\|f\|_{\dot{B}_{p, q}^{s}}:=\left\|\left(2^{s j}\left\|\Delta_{j} f\right\|_{L^{p}}\right)_{j \in \mathbb{Z}}\right\|_{\ell^{q}}
$$

is finite. It is known and not difficult to check that this definition is independent of the initial choice of the function $\varphi$ with the above mentioned property, the resulting norms being equivalent. The condition that $s<\frac{d}{p}$ is necessary to ensure the fact that we have defined a proper Banach space (otherwise convergence should be interpreted in $\mathcal{S}^{\prime} / \Pi_{n}$ where $\Pi_{n}$ is the space of polynomials of total degree $n=\left\lfloor s-\frac{d}{p}\right\rfloor$, for more details see [8] and the discussion therein p. 153-155).

We shall consider Besov spaces of regularity index $s$ which is either positive or negative. Using discrete Hardy inequalities, it is also possible to characterize these spaces using only the operator $S_{j}:$ for $s>0$, we have

$$
\|f\|_{\dot{B}_{p, q}^{s}} \sim\left\|\left(2^{s j}\left\|\left(I-S_{j}\right) f\right\|_{L^{p}}\right)_{j \in \mathbb{Z}}\right\|_{\ell^{q}}
$$

and for $s<0$,

$$
\|f\|_{\dot{B}_{p, q}^{s}} \sim\left\|\left(2^{s j}\left\|S_{j} f\right\|_{L^{p}}\right)_{j \in \mathbb{Z}}\right\|_{\ell^{q}} .
$$

Equivalent norms for Besov spaces can also be obtained with the discrete frequencies $2^{j}$ replaced by a continuous one: introducing for any $\lambda>0$ the operators

$$
S_{\lambda} f=\lambda^{d} \varphi(\lambda \cdot) * f \text { and } \Delta_{\lambda} f=S_{2 \lambda} f-S_{\lambda} f=\lambda^{d} \psi(\lambda \cdot) * f
$$

we then find that $\|f\|_{\dot{B}_{p, q}^{s}}$ is equivalent to the norm of the function

$$
F(\lambda):=\lambda^{s}\left\|\Delta_{\lambda} f\right\|_{L^{p}}
$$

in $L^{q}\left(\mathbb{R}_{+}, \frac{d t}{t}\right)$. By similar arguments as when working with discrete frequency, we can equivalently choose

$$
F(\lambda):=\lambda^{s}\left\|\left(I-S_{\lambda}\right) f\right\|_{L^{p}}
$$

for $s>0$ or

$$
F(\lambda):=\lambda^{s}\left\|S_{\lambda} f\right\|_{L^{p}}
$$

for $s<0$. For the purpose of our analysis, we shall also need estimates on the derivative $F^{\prime}(\lambda)=\frac{d F}{d \lambda}$, as expressed by the following result.

Lemma 2.1 There exists a constant $K$ which depends only on $s, d, p, q$ and the choice of $\varphi$ such that for all $f \in \dot{B}_{p, q}^{s}$, the function $G(\lambda):=\lambda F^{\prime}(\lambda)$ belongs to $L^{q}\left(\mathbb{R}_{+}, \frac{d t}{t}\right)$ with

$$
\|G\|_{L^{q}\left(\mathbb{R}_{+}, \frac{d t}{t}\right)} \leq K\|f\|_{\dot{B}_{p, q}^{s}},
$$

where $F$ is defined by (2.7), or by (2.8) when $s>0$, or by (2.9) when $s<0$.

Proof: we only consider the case where $F$ is defined by (2.7), the two others being treated in the same way. We first estimate the derivative of the function $g(\lambda):=\left\|\Delta_{\lambda} f\right\|_{L^{p}}$. Clearly, we have

$$
\left|g^{\prime}(\lambda)\right| \leq\left\|\frac{d}{d \lambda}\left(\lambda^{d} \psi(\lambda \cdot)\right) * f\right\|_{L^{p}}=\left\|\lambda^{d-1} \tilde{\psi}(\lambda \cdot) * f+d \lambda^{d-1} \psi(\lambda \cdot) * f\right\|_{L^{p}},
$$


with $\tilde{\psi}(x)=x \cdot \nabla \psi(x)$. Since we have

$$
F^{\prime}(\lambda)=s \lambda^{s-1} g(\lambda)+\lambda^{s} g^{\prime}(\lambda),
$$

it follows that

$$
|G(\lambda)| \leq(s+d) F(\lambda)+\lambda^{s}\left\|\lambda^{d} \tilde{\psi}(\lambda \cdot) * f\right\|_{L^{p}}:=(s+d) F(\lambda)+\widetilde{F}(\lambda)
$$

The function $\tilde{\psi}$ satisfies $\widehat{\tilde{\psi}}(\omega)=\widehat{\tilde{\varphi}}\left(\frac{\omega}{2}\right)-\widehat{\tilde{\varphi}}(\omega)$ with

$$
\widehat{\tilde{\varphi}}(\omega):=\hat{\varphi}(\omega)+\omega \cdot \nabla \hat{\varphi}(\omega) .
$$

Since $\hat{\tilde{\varphi}}$ is $C^{\infty}$, compactly supported in the ball $\{|\omega| \leq M\}$, and such that $\hat{\varphi}(\omega)=1$ if $|\omega| \leq 1$, we obtain from the definition of Besov spaces that

$$
\|G\|_{L^{q}\left(\mathbb{R}_{+}, \frac{d t}{t}\right)} \leq(s+d)\|F\|_{L^{q}\left(\mathbb{R}_{+}, \frac{d t}{t}\right)}+\|\widetilde{F}\|_{L^{q}\left(\mathbb{R}_{+}, \frac{d t}{t}\right)} \leq K\|f\|_{\dot{B}_{p, q}^{s},},
$$

for some $K$ which depends on $(s, d, p, q, \varphi)$.

\section{Proof of the refined inequality}

We first observe that we may prove (1.3) in the particulary case where $f$ is a $C^{\infty}$ compactly supported function, and derive by density the general validity of the inequality.

We decompose $f$ into low and high frequencies according to

$$
f=S_{\lambda} f+\left(I-S_{\lambda}\right) f
$$

where $S_{\lambda}$ is the convolution operator defined in the previous section. From the characterization of Besov spaces which was recalled in $\S 2.2$, we have

$$
\int_{0}^{\infty}[F(\lambda)]^{q} \frac{d \lambda}{\lambda} \leq C_{0}\|f\|_{\dot{B}_{\infty, q}^{s-\frac{d}{q}}}^{q}
$$

where $C_{0}$ is an absolute constant and

$$
F(\lambda):=\lambda^{s-\frac{d}{q}}\left\|S_{\lambda} f\right\|_{L^{\infty}} .
$$

We also have

$$
\int_{0}^{+\infty} \lambda^{q s}\left\|\left(I-S_{\lambda}\right) f\right\|_{L^{q}}^{q} \frac{d \lambda}{\lambda} \leq C_{1}\|f\|_{\dot{B}_{q, q}^{s}}^{q},
$$

where $C_{1}$ is also an absolute constant.

We now consider the function $\lambda \mapsto t(\lambda)$ defined by

$$
t(\lambda)=2 \lambda^{\frac{d}{p}} F(\lambda)=2 \lambda^{s+\frac{d}{p}-\frac{d}{q}}\left\|S_{\lambda} f\right\|_{L^{\infty}}=2\left\|S_{\lambda} f\right\|_{L^{\infty}} .
$$

This function may not be monotone but it tends towards 0 as $\lambda \rightarrow 0$ and towards $M:=2\|f\|_{L^{\infty}} \geq\|f\|_{L^{\infty}}$ as $\lambda \rightarrow+\infty$. We may thus use it as a change of variable in the expression (2.6) of the $L^{p, q}$ quasi-norm, which yields

$$
\|f\|_{L^{p, q}}^{q}=p \int_{0}^{+\infty} t^{q} \mu_{f}(t)^{\frac{q}{p}} d t=p \int_{0}^{2 M} t^{q} \mu_{f}(t)^{\frac{q}{p}} d t=p \int_{0}^{+\infty} t(\lambda)^{q-1} t^{\prime}(\lambda)\left(\mu_{f}(t(\lambda))\right)^{\frac{q}{p}} d \lambda .
$$

From the triangle inequality we have

$$
\{x ;|f(x)|>t\} \subset\left\{x ;\left|S_{\lambda} f(x)\right|>\frac{t}{2}\right\} \cup\left\{x ;\left|\left(I-S_{\lambda}\right) f(x)\right|>\frac{t}{2}\right\}
$$

Since we have $\left|S_{\lambda} f(x)\right| \leq \frac{t(\lambda)}{2}$ for all $x$, it follows that

$$
\{x ;|f(x)|>t(\lambda)\} \subset\left\{x ;\left|\left(I-S_{\lambda}\right) f\right|>\frac{t(\lambda)}{2}\right\},
$$


i.e. $\mu_{f}(t(\lambda)) \leq \mu_{\left(I-S_{\lambda}\right) f}\left(\frac{t(\lambda)}{2}\right)$. Combining this observation together with (3.11), we obtain

$$
\begin{aligned}
& \|f\|_{L^{p, q}}^{q} \leq p \int_{0}^{+\infty} t(\lambda)^{q-1} t^{\prime}(\lambda)\left[\mu_{\left(I-S_{\lambda}\right) f}\left(\frac{t(\lambda)}{2}\right)\right]^{\frac{q}{p}} d \lambda \\
& =2^{q} d \int_{0}^{+\infty}\left[\lambda^{\frac{d}{p}} F(\lambda)\right]^{q-1}\left(\lambda^{\frac{d}{p}-1} F(\lambda)\right)\left[\mu_{\left(I-S_{\lambda}\right) f}\left(\frac{t(\lambda)}{2}\right)\right]^{\frac{q}{p}} d \lambda \\
& +2^{q} p \int_{0}^{+\infty}\left[\lambda^{\frac{d}{p}} F(\lambda)\right]^{q-1}\left(\lambda^{\frac{d}{p}} F^{\prime}(\lambda)\right)\left[\mu_{\left(I-S_{\lambda}\right) f}\left(\frac{t(\lambda)}{2}\right)\right]^{\frac{q}{p}} d \lambda \\
& =2^{q} d \int_{0}^{+\infty} \lambda^{\frac{d q}{p}-1} F(\lambda)^{q}\left[\mu_{\left(I-S_{\lambda}\right) f}\left(\frac{t(\lambda)}{2}\right)\right]^{\frac{q}{p}} d \lambda \\
& +2^{q} p \int_{0}^{+\infty} \lambda^{\frac{d q}{p}} F(\lambda)^{q-1} F^{\prime}(\lambda)\left[\mu_{\left(I-S_{\lambda}\right) f}\left(\frac{t(\lambda)}{2}\right)\right]^{\frac{q}{p}} d \lambda \\
& :=T_{0}+T_{1}
\end{aligned}
$$

In order to estimate further the two above terms, we remark that

$$
\mu_{\left(I-S_{\lambda}\right) f}\left(\frac{t(\lambda)}{2}\right) \leq\left(\frac{t(\lambda)}{2}\right)^{-q}\left\|\left(I-S_{\lambda}\right) f\right\|_{L^{q}}^{q}=\left(\lambda^{\frac{d}{p}} F(\lambda)\right)^{-q}\left\|\left(I-S_{\lambda}\right) f\right\|_{L^{q}}^{q} .
$$

For $T_{0}$, this gives

$$
\begin{aligned}
T_{0} & \leq 2^{q} d \int_{0}^{+\infty} \lambda^{\frac{d q}{p}-1} F(\lambda)^{q}\left[\left(\lambda^{\frac{d}{p}} F(\lambda)\right)^{-q}\left\|\left(I-S_{\lambda}\right) f\right\|_{L^{q}}^{q}\right]^{\frac{q}{p}} d \lambda \\
& =2^{q} d \int_{0}^{+\infty} F(\lambda)^{q\left(1-\frac{q}{p}\right)}\left[\lambda^{d-\frac{d q}{p}}\left\|\left(I-S_{\lambda}\right) f\right\|_{L^{q}}^{q}\right]^{\frac{q}{p}} \frac{d \lambda}{\lambda} \\
& =2^{q} d \int_{0}^{+\infty} F(\lambda)^{q\left(1-\frac{q}{p}\right)}\left[\lambda^{q s}\left\|\left(I-S_{\lambda}\right) f\right\|_{L^{q}}^{q}\right]^{\frac{q}{p}} \frac{d \lambda}{\lambda} .
\end{aligned}
$$

Applying Hölder inequality, we thus obtain

$$
\begin{aligned}
T_{0} & \leq 2^{q} d\left(\int_{0}^{+\infty} F(\lambda)^{q} \frac{d \lambda}{\lambda}\right)^{1-\frac{q}{p}}\left(\int_{0}^{+\infty} \lambda^{q s}\left\|\left(I-S_{\lambda}\right) f\right\|_{L^{q}}^{q} \frac{d \lambda}{\lambda}\right)^{\frac{q}{p}} \\
& \leq 2^{q} d\left(\int_{0}^{+\infty} F(\lambda)^{q} \frac{d \lambda}{\lambda}\right)^{\frac{q s}{d}}\left(\int_{0}^{+\infty} \lambda^{q s}\left\|\left(I-S_{\lambda}\right) f\right\|_{L^{q}}^{q} \frac{d \lambda}{\lambda}\right)^{1-\frac{q s}{d}} \\
& \leq C\left(\|f\|_{\dot{B}_{\infty, q}^{s-\frac{d}{q}}}^{\frac{q s}{d}}\|f\|_{\dot{B}_{q, q}^{s}}^{1-\frac{q s}{d}}\right)^{q} .
\end{aligned}
$$

where $C$ is an absolute constant. Proceeding in an exactly similar manner for $T_{1}$, we obtain

$$
T_{1} \leq 2^{q} p\left(\int_{0}^{+\infty} F(\lambda)^{q-1} \lambda F^{\prime}(\lambda) \frac{d \lambda}{\lambda}\right)^{\frac{q s}{d}}\left(\int_{0}^{+\infty} \lambda^{q s}\left\|\left(I-S_{\lambda}\right) f\right\|_{L^{q}}^{q} \frac{d \lambda}{\lambda}\right)^{1-\frac{q s}{d}} .
$$

Using Lemma 2.1 together with a Hölder inequality, we see that the integral in the first factor can again be controlled by $\|f\|_{\dot{B}_{\infty, q}^{s-\frac{d}{q}}}^{q}$, and therefore we also obtain

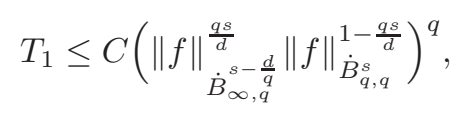

where $C$ is an absolute constant. Combining our estimates for $T_{0}$ and $T_{1}$ we have proved that

$$
\|f\|_{L^{p, q}}^{q} \leq C\left(\|f\|_{\dot{B}_{\infty, q}^{s-\frac{d}{q}}}^{\frac{q s}{d}}\|f\|_{\dot{B}_{q, q}^{s}}^{1-\frac{q s}{d}}\right)^{q},
$$

which is the desired result.

Remark 3.1 For our range of indices $(s, p, q)$, we also have a continuous embedding of $\dot{B}_{q, r}^{s}$ into $L^{p, r}$ for any $1 \leq r \leq \infty$ (this can easily be proved from the standard Sobolev embedding by a real interpolation argument). This suggests a correponding improved inequality of the form

$$
\|f\|_{L^{p, r}} \leq C\|f\|_{\dot{B}_{q, r}^{s}}^{1-\frac{q s}{d}}\|f\|_{\dot{B}_{\infty, r}^{s-\frac{d}{q}}}^{\frac{q s}{d}},
$$

but the proof of this does not seem obvious to us, neither by interpolation arguments, nor by an adaptation of our current proof (that corresponds to the case $r=q$ ). 


\section{Sharpness of the inequality}

We shall discuss the sharpness of (1.3) through two examples. The first one shows that the refined estimates are sharp with respect to oscillating functions: consider for all positive $\omega$, the function

$$
\varphi_{\omega}(x)=e^{i \omega x_{1}} \varphi(x)
$$

where $\varphi$ is a fixed function in $\mathcal{S}\left(\mathbb{R}^{d}\right)$. For such a function, $\left\|\varphi_{\omega}\right\|_{L^{p, q}}$ is independent of $\omega$ since the $L^{p, q}$ quasi-norm only depends on the modulus. On the other hand for $|\omega|>1$,

$$
\left\|\varphi_{\omega}\right\|_{\dot{B}_{q, q}^{s}} \sim \omega^{s}
$$

Therefore, the Sobolev inequality (1.2) is not sharp when $\omega$ is large. It is also easily checked that for $|\omega|>1$

$$
\left\|\varphi_{\omega}\right\|_{\substack{\dot{B}_{\infty}-\frac{d}{q} \\ \text { d }}} \sim \omega^{s-\frac{d}{q}}
$$

(see [1] for the proof of these equivalences) and therefore

$$
\left\|\varphi_{\omega}\right\|_{\dot{B}_{q, q}^{s}}^{1-\frac{q s}{d}}\left\|\varphi_{\omega}\right\|_{\dot{B}_{\infty, q}^{s-d / q}}^{\frac{q s}{d}} \sim \omega^{s\left(1-\frac{q s}{d}\right)+\left(s-\frac{d}{q}\right) \frac{q s}{d}}=1
$$

which shows that (1.3) is sharp for such oscillatory functions.

The defect of this first example is that it does not make much distinction between (1.3) and the improved Sobolev inequality (1.1) in $L^{p}$ spaces : indeed, we also have that $\left\|\varphi_{\omega}\right\|_{L^{p}}$ is independent of $\omega$ and that $\left\|\varphi_{\omega}\right\|_{\dot{B}_{\infty, \infty}^{s-\frac{d}{q}}} \sim \omega^{s-\frac{d}{q}}$. In particular, this example does not reveal the necessity of using $\dot{B}_{\infty, q}^{s-\frac{d}{q}}$ in place of $\dot{B}_{\infty, \infty}^{s-\frac{d}{q}}$ when we want to control the $L^{p, q}$ quasi-norm. A finer example is needed order to show that this cannot be avoided. This example should contain oscillations in a wide range of frequencies in order to make the distinction between $\dot{B}_{\infty, q}^{s-\frac{d}{q}}$ and $\dot{B}_{\infty, \infty}^{s-\frac{d}{q}}$, whereas it should also contain a wide range of magnitudes in order to distinguish between $L^{p}$ and $L^{p, q}$. Our example has roughly the form of a hyperbolic "chirp"

$$
f(x)=x^{-\alpha} \sin \left(\frac{1}{x}\right)
$$

with $\alpha>0$. For the sake of simplicity, we have placed ourselves in the one-dimensional case $d=1$ although this example can be generalized to arbitrary dimensions. In order to simplify our analysis, we shall define a variant of $f$ in the form of a wavelet series. We recall that the Haar system is defined by the functions

$$
\psi_{j, k}(x)=2^{\frac{j}{2}} \psi\left(2^{j} x-k\right), \quad j, k \in \mathbb{Z},
$$

where $\psi:=\chi_{\left[0, \frac{1}{2}[\right.}-\chi_{\left[\frac{1}{2}, 1\right]}$. Besov spaces $\dot{B}_{p, q}^{s}$ can be characterized as follows by wavelets (see e.g. [15] or $[6])$ : if $f=\sum_{j, k} d_{j, k} \psi_{j, k}$, then

$$
\|f\|_{\dot{B}_{p, q}^{s}} \sim\left\|\left(2^{\left(s+\frac{1}{2}-\frac{1}{p}\right) j}\left\|\left(d_{j, k}\right)_{k \in \mathbb{Z}}\right\|_{\ell^{p}}\right)_{j \in \mathbb{Z}}\right\|_{\ell^{q}}
$$

In the case of the Haar system, this characterisation holds if $\frac{1}{p}-1<s<\frac{1}{p}$ (due to the lack of smoothness of the functions $\psi_{j, k}$ ) which is sufficient for our purpose.

Let $q \geq 1$ and $0<s<\frac{1}{q}$, and let $\frac{1}{p}:=\frac{1}{q}-s$. For $J>0$, we consider the function

$$
f_{J}(x)=\sum_{j=1}^{J} 2^{\left(\frac{1}{p}-\frac{1}{2}\right) j} \psi_{j, 1}(x)=\sum_{j=1}^{J} 2^{\frac{j}{p}} \psi\left(2^{j} x-1\right) .
$$

Note that the wavelets in the expansion of $f_{J}$ have disjoint supports. We first notice that we have

$$
\left\|f_{J}\right\|_{\dot{B}_{q, q}^{s}} \sim\left(\sum_{j=1}^{J} 2^{\left(q s+\frac{q}{2}-1\right) j} 2^{\left(\frac{q}{p}-\frac{q}{2}\right) j}\right)^{\frac{1}{q}}=J^{\frac{1}{q}} .
$$


For the Besov norms of negative indices, we have

$$
\left\|f_{J}\right\|_{\dot{B}_{\infty, \infty}^{s-\frac{1}{q}}} \sim \sup _{j=1}^{J} 2^{\left(s-\frac{1}{q}+\frac{1}{2}\right) j} 2^{\left(\frac{1}{p}-\frac{1}{2}\right) j}=1
$$

and

$$
\left\|f_{J}\right\|_{\substack{\dot{B}_{\infty}-\frac{1}{q} \\ \text { s. }}} \sim\left(\sum_{j=1}^{J}\left[2^{\left(s-\frac{1}{q}+\frac{1}{2}\right) j} 2^{\left(\frac{1}{p}-\frac{1}{2}\right) j}\right]^{q}\right)^{\frac{1}{q}}=J^{\frac{1}{q}} .
$$

Since the wavelets in the expansion of $f_{J}$ have disjoint supports, the $L^{p}$ norm is of the order

$$
\left\|f_{J}\right\|_{L^{p}}=\left(\sum_{j=1}^{J}\left\|2^{\frac{j}{p}} \psi\left(2^{j} \cdot-1\right)\right\|_{L^{p}}^{p}\right)^{\frac{1}{p}} \sim J^{1 / p} .
$$

Remarking that the rearrangement function of $f_{J}$ is simply given by

$$
f_{J}^{*}(s)=\left|f_{J}\left(s+2^{-J}\right)\right|
$$

we can also easily evaluate its $L^{p, q}$ quasi-norm using (2.5) which gives

$$
\begin{aligned}
\left\|f_{J}\right\|_{L^{p, q}} & =\left(\sum_{j=1}^{J} 2^{\frac{j q}{p}} \int_{0}^{2^{-j}}\left(s+2^{-j}-2^{-J}\right)^{\frac{q}{p}-1} d s\right)^{\frac{1}{q}} \\
& \sim\left(\sum_{j=1}^{J} 2^{\frac{j q}{p}} 2^{-j} 2^{-j\left(\frac{q}{p}-1\right)}\right)^{\frac{1}{q}} \\
& =J^{\frac{1}{q}} .
\end{aligned}
$$

From all these estimates, we obtain that

$$
\left\|f_{J}\right\|_{\dot{B}_{q, q}^{s}}^{1-q s}\left\|f_{J}\right\|_{\substack{\dot{B}_{\infty, \infty}^{s-\frac{1}{q}} \\ q s}}^{q} \sim J^{\frac{1}{q}-s}=J^{\frac{1}{p}} \sim\left\|f_{J}\right\|_{L^{p}}
$$

and

$$
\left\|f_{J}\right\|_{\dot{B}_{q, q}^{s}}^{1-q s}\left\|f_{J}\right\|_{\dot{B}_{\infty, q}^{s-\frac{1}{q}}}^{q s} \sim J^{\frac{1}{q}-s} J^{s}=J^{\frac{1}{q}} \sim\left\|f_{J}\right\|_{L^{p, q}} .
$$

Again (1.3) and (1.1) are sharp, but this example also shows that the use of $\dot{B}_{\infty, q}^{s-\frac{d}{q}}$ in place of $\dot{B}_{\infty, \infty}^{s-\frac{d}{q}}$ is unavoidable for the validity of (1.3).

\section{Refined Hardy inequalities}

Let as in the previous sections $1<p<\infty$ and $s \in] 0, \frac{d}{q}\left[\right.$, with $\frac{1}{p}=\frac{1}{q}-\frac{s}{d}$. For such indices, we claim that the refined Hardy inequality (1.4) holds. It is actually a direct consequence of (1.3) combined with a generalized version of Hölder's inequality known as O'Neil inequalities : if $1 \leq p_{1}, p_{2}, q_{1}, q_{2} \leq \infty$, then for any $f \in L^{p_{1}, q_{1}}\left(\mathbb{R}^{d}\right)$ and $g \in L^{p_{2}, q_{2}}\left(\mathbb{R}^{d}\right)$,

$$
\|f g\|_{L^{p, q}\left(\mathbb{R}^{d}\right)} \leq C\|f\|_{L^{p_{1}, q_{1}\left(\mathbb{R}^{d}\right)}}\|g\|_{L^{p_{2}, q_{2}\left(\mathbb{R}^{d}\right)}},
$$

where $\frac{1}{p}=\frac{1}{p_{1}}+\frac{1}{p_{2}}$ and $\frac{1}{q}=\frac{1}{q_{1}}+\frac{1}{q_{2}}$ and $C=C\left(p_{1}, p_{2}, q_{1}, q_{2}\right)$ (a first proof of these inequalities using rearrangements can be found in [17], while a more modern proof would proceed by bilinear interpolation between the usual inequalities for Lebesgue spaces).

We now take $g(x)=\frac{1}{|x|^{s}}$ and apply (5.13), in the specific form

$$
\|f g\|_{L^{q, q}\left(\mathbb{R}^{d}\right)} \leq C\|f\|_{L^{p, q}\left(\mathbb{R}^{d}\right)}\|g\|_{L^{r, \infty}\left(\mathbb{R}^{d}\right)}
$$

where $\frac{1}{q}=\frac{1}{p}+\frac{1}{r}$, i.e. $r=\frac{d}{s}$. Since obviously $g \in L^{r, \infty}\left(\mathbb{R}^{d}\right)$, we have

$$
\left(\int \frac{|f(x)|^{q}}{|x|^{s q}}\right)^{\frac{1}{q}} \leq C\|f\|_{L^{p, q}\left(\mathbb{R}^{d}\right)} .
$$


Combining this with (1.3), we obtain (1.4). Let us mention that this inequality is obtained in [1] for $q=2$ by a different method which can not be generalized to the general $L^{q}$ framework.

Concerning the sharpness of (1.4), we can make similar observations as for (1.3). In particular, this inequality is sharp for oscillatory functions of the type $\varphi_{\omega}(x)=e^{i \omega x_{1}} \varphi(x)$. It is also easily checked that the sequence $f_{J}$ defined by (4.12) satisfies

$$
\left(\int \frac{\left|f_{J}(x)\right|^{q}}{|x|^{s q}}\right)^{\frac{1}{q}} \sim J^{\frac{1}{q}} \sim\left\|f_{J}\right\|_{L^{p, q\left(\mathbb{R}^{d}\right)}},
$$

making (1.4) sharp for such functions, and justifying the use of $\dot{B}_{\infty, q}^{s-\frac{d}{q}}$ in place of $\dot{B}_{\infty, \infty}^{s-\frac{d}{q}}$.

\section{References}

[1] H. Bahouri, J.-Y. Chemin and I. Gallagher, Refined Hardy inequalities, Annali della Scuola Normale di Pisa (5) 375-391, 2006.

[2] C. Bennett and R. Sharpley, Interpolation of operators, Academic Press, 1988.

[3] A. Cohen, Y. Meyer and F. Oru, Improved Sobolev inequalities, Proceedings séminaires X-EDP, Ecole Polytechnique, 91128 Palaiseau, France,1998.

[4] A. Cohen, W. Dahmen, I. Daubechies and R. DeVore, Harmonic analysis of the space BV, Revista Mathematica Iberoamericana 19, 235-263, 2003.

[5] J. Bergh and J. Löfström, Interpolation soaces. An introduction, Springer-Verlag, Berlin, 1976.

[6] A. Cohen, Numerical analysis of wavelet methods, Elsevier, 2003.

[7] R. DeVore and G. Lorentz, Constructive Approximation, Springer Verlag, 1993.

[8] M. Frazier and B. Jawerth, A discrete transform and decompositions of distribution spaces, J. of Functional Analysis 93, 34-170, 1990.

[9] P. Gérard, Description du défaut de compacité de l'injection de Sobolev, ESAIM. Control, Optimisation and Calculus of Variations, 3, 213-233, 1998.

[10] P. Gérard, Y. Meyer and F. Oru, Inégalités de Sobolev précisées, Séminaire X-EDP, École Polytechnique, 1996.

[11] S. Jaffard, Analysis of the lack of compactness in the critical Sobolev inbeddings, J. Functional Analysis 161, 384-396, 1999.

[12] S. Jaffard and Y. Meyer, Wavelet methods for pointwise regularity and local oscillations of functions, Memoirs of the AMS, Vol.123, no. 587, 1996.

[13] M. Ledoux, On improved Sobolev embedding theorems, Mathematical Research Letters 10, 659-669, 2003.

[14] J. Martin and M. Milman, Sharp Gagliardo-Nirenberg inequalities via Symmetrization, Mathematical Research Letters 14, 49-62, 2007.

[15] Y. Meyer, Ondelettes et opérateurs, Hermann, 1990.

[16] Y. Meyer, Oscillating Patterns in some Nonlinear Evolution Equations. Mathematical Foundation of Turbulent Viscous Flows, Lecture Notes in Mathematics 1871, Springer, 2006.

[17] R. O'Neil, Convolution operators on L(p,q) spaces, Duke Math.J. (30) 129-142, 1963.

[18] H. Triebel, Interpolation theory, function spaces, differential operators, North-Holland, Amsterdam, 1978. 
Hajer Bahouri

Centre de Mathématiques - Faculté de Sciences et Technologie

Université Paris XII - Val de Marne

61, avenue du Général de Gaulle

94010 Creteil Cedex, France

bahouri@univ-paris12.fr

\section{Albert Cohen}

UPMC Univ Paris 06, UMR 7598, Laboratoire Jacques-Louis Lions, F-75005, Paris, France CNRS, UMR 7598, Laboratoire Jacques-Louis Lions, F-75005, Paris, France

cohen@ann.jussieu.fr 